\title{
OP-1 Compared with Iliac Crest Autograft in Instrumented Posterolateral Fusion
}

\author{
A Randomized, Multicenter Non-Inferiority Trial \\ Diyar Delawi, MD, PhD, Wilco Jacobs, MSc, PhD, Job L.C. van Susante, MD, PhD, Ludovic Rillardon, MD, \\ Domenico Prestamburgo, MD, Nicola Specchia, MD, Emmanuel Gay, MD, Nico Verschoor, MD, \\ Carlos Garcia-Fernandez, MD, Enrique Guerado, MD, Henriette Quarles van Ufford, MD, PhD, \\ Moyo C. Kruyt, MD, PhD, Wouter J.A. Dhert, MD, PhD, and F. Cumhur Oner, MD, PhD
}

\begin{abstract}
Background: Spinal fusion with the use of autograft is a commonly performed procedure. However, harvesting of bone from the iliac crest is associated with complications. Bone morphogenetic proteins (BMPs) are extensively used as alternatives, often without sufficient evidence of safety and efficacy. The purpose of this study was to investigate non-inferiority of osteogenic protein-1 (OP-1, also known as BMP-7) in comparison with iliac crest bone graft in posterolateral fusions.
\end{abstract}

Methods: This study was a randomized, controlled multicenter trial. Patients who underwent a single-level instrumented posterolateral fusion of the lumbar spine for degenerative or isthmic spondylolisthesis with symptoms of neurological compression were randomized to receive OP-1 combined with local bone (OP-1 group) or autologous bone graft from the iliac crest combined with local bone (autograft group). The primary outcome was overall success, defined as a combination of clinical success and evidence of fusion on computed tomography (CT) scans, at one year postoperatively.

Results: One hundred and nineteen patients were included in the study, and analysis of the overall outcome was performed for 113. Non-inferiority of OP-1 compared with iliac crest autograft was not found at one year, with a success rate of $40 \%$ in the OP-1 group versus $54 \%$ in the autograft group (risk difference $=-13.3 \%, 90 \%$ confidence interval $[\mathrm{Cl}]=-28.6 \%$ to $+2.10 \%$ ). This was due to the lower rate of fusion (the primary aim of OP-1 application) seen on the CT scans in the OP-1 group (54\% versus $74 \%$ in the autograft group, $p=0.03$ ). There were no adverse events that could be directly related to the use of OP-1.

Conclusions: OP-1 with a collagen carrier was not as effective as autologous iliac crest bone for achieving fusion and cannot be recommended in instrumented posterolateral lumbar fusion procedures.

Level of Evidence: Therapeutic Level I. See Instructions for Authors for a complete description of levels of evidence.

Peer Review: This article was reviewed by the Editor-in-Chief and one Deputy Editor, and it underwent blinded review by two or more outside experts. The Deputy Editor reviewed each revision of the article, and it underwent a final review by the Editor-in-Chief prior to publication. Final corrections and clarifications occurred during one or more exchanges between the author(s) and copyeditors.

S pinal fusion surgery is frequently performed for several indications ${ }^{1}$. The current practice is to use autologous bone from the iliac crest to create an osseous bridge between spinal segments. Harvesting of bone graft requires an additional surgical procedure, which is associated with potential complications $\mathrm{s}^{2-5}$.
Induction of bone regeneration with bioactive molecules is a potential alternative to the use of autologous bone. The field was launched by Urist, who identified proteins from the bone matrix responsible for ectopic bone induction, which he called bone morphogenetic proteins (BMPs) $)^{6}$. Currently, two BMPs are commercially available: BMP-2 (InFuse; Medtronic) and BMP-7

Disclosure: One or more of the authors received payments or services, either directly or indirectly (i.e., via his or her institution), from a third party in support of an aspect of this work. In addition, one or more of the authors, or his or her institution, has had a financial relationship, in the thirty-six months prior to submission of this work, with an entity in the biomedical arena that could be perceived to influence or have the potential to influence what is written in this work. No author has had any other relationships, or has engaged in any other activities, that could be perceived to influence or have the potential to influence what is written in this work. The complete Disclosures of Potential Conflicts of Interest submitted by authors are always provided with the online version of the article. 
The Journal of Bone \& Joint Surgery Jbjs.org Volume 98-A · Number 6 - March 16, 2016
OP-1 Compared with Iliac Crest Autograft in

Instrumented Posterolateral Fusion

\section{TABLE I Inclusion and Exclusion Criteria}

\begin{tabular}{|ll|}
\hline \multicolumn{2}{|c|}{ Inclusion Criteria } \\
\hline $\begin{array}{l}\text { Degenerative or isthmic spondylolisthesis (Grades I and II*) with } \\
\text { central or foraminal stenosis }\end{array}$ & Gross instability requiring multiple levels of fusion \\
$\begin{array}{l}\text { Eligible for decompression and single-level fusion (L3-S1) } \\
\text { Symptoms of radiculopathy and/or neurogenic claudication }\end{array}$ & Severe osteoporosis or osteopenia \\
Preoperative ODI $>30$ & Suspicion of active spinal or systemic infection \\
Not responsive to $\geq 6$ mo of nonoperative treatment & Pregnancy or a plan to become pregnant \\
No previous fusion attempt(s) at affected level & Known sensitivity to collagen \\
Skeletally mature & Morbid obesity \\
Informed consent & Use of systemic corticosteroids during previous yr \\
\hline *According to the Meyerding classification ${ }^{34}$. & Known to require additional surgery to lumbar spinal region within 6 mo \\
\hline
\end{tabular}

(Osigraft and OP-1 Putty; Stryker), also known as osteogenic protein-1 (OP-1). The U.S. Food and Drug Administration (FDA) approved the use of BMP-2 for a spinal indication, restricted to anterior lumbar interbody fusion with a specific cage ${ }^{7}$, and OP-1 has only a Humanitarian Device Exemption approval for revision of posterolateral lumbar fusion in compromised patients ${ }^{8}$. Despite the limited approval, BMP usage has been rapidly incorporated into the standard surgical practice, with use in nearly $40 \%$ of lumbosacral spinal fusions performed in the U.S .

The scientific basis for the use of BMP-2 for spinal indications has been criticized because of allegations of methodological biases and structural underreporting of adverse events in industry-sponsored publications ${ }^{10-14}$. There is currently no indisputable evidence of the efficacy of OP-1 in spinal applications.

In the present non-inferiority study, we compared OP-1 with autologous bone in single-level instrumented lumbar posterolateral fusion. Non-inferiority of OP-1 would make OP-1 a valuable alternative circumventing bone-graft-related morbidity.

\section{Materials and Methods}

Study Design

This randomized, multicenter non-inferiority trial of patients requiring single1 level fusion of the lumbar spine was an investigator-initiated study performed with an unconditional grant from the manufacturer of OP-1 (Stryker). The trial

TABLE II Demographic and Clinical Details

\begin{tabular}{|c|c|c|c|}
\hline & OP-1 Group (N = 60) & Autograft Group ( $N=59$ ) & P Value \\
\hline Age* $(y r)$ & $54 \pm 14$ & $55 \pm 13$ & 0.65 \\
\hline Body mass index* $\left(\mathrm{kg} / \mathrm{m}^{2}\right)$ & $26.6 \pm 4$ & $25.2 \pm 5$ & 0.17 \\
\hline Sex† & & & 0.46 \\
\hline Male & 27 (45\%) & 25 (42\%) & \\
\hline Female & 33 (55\%) & $34(58 \%)$ & \\
\hline Origin of instability $\dagger$ & & & 0.71 \\
\hline Degenerative spondylolisthesis & $31(52 \%)$ & $33(56 \%)$ & \\
\hline Isthmic spondylolisthesis & $29(48 \%)$ & $26(44 \%)$ & \\
\hline Level fused $\dagger$ & & & 0.16 \\
\hline L3-L4 & 9 (15\%) & $6(10 \%)$ & \\
\hline Preoperative ODI*キ & $44 \pm 16$ & $44 \pm 14$ & 0.97 \\
\hline
\end{tabular}




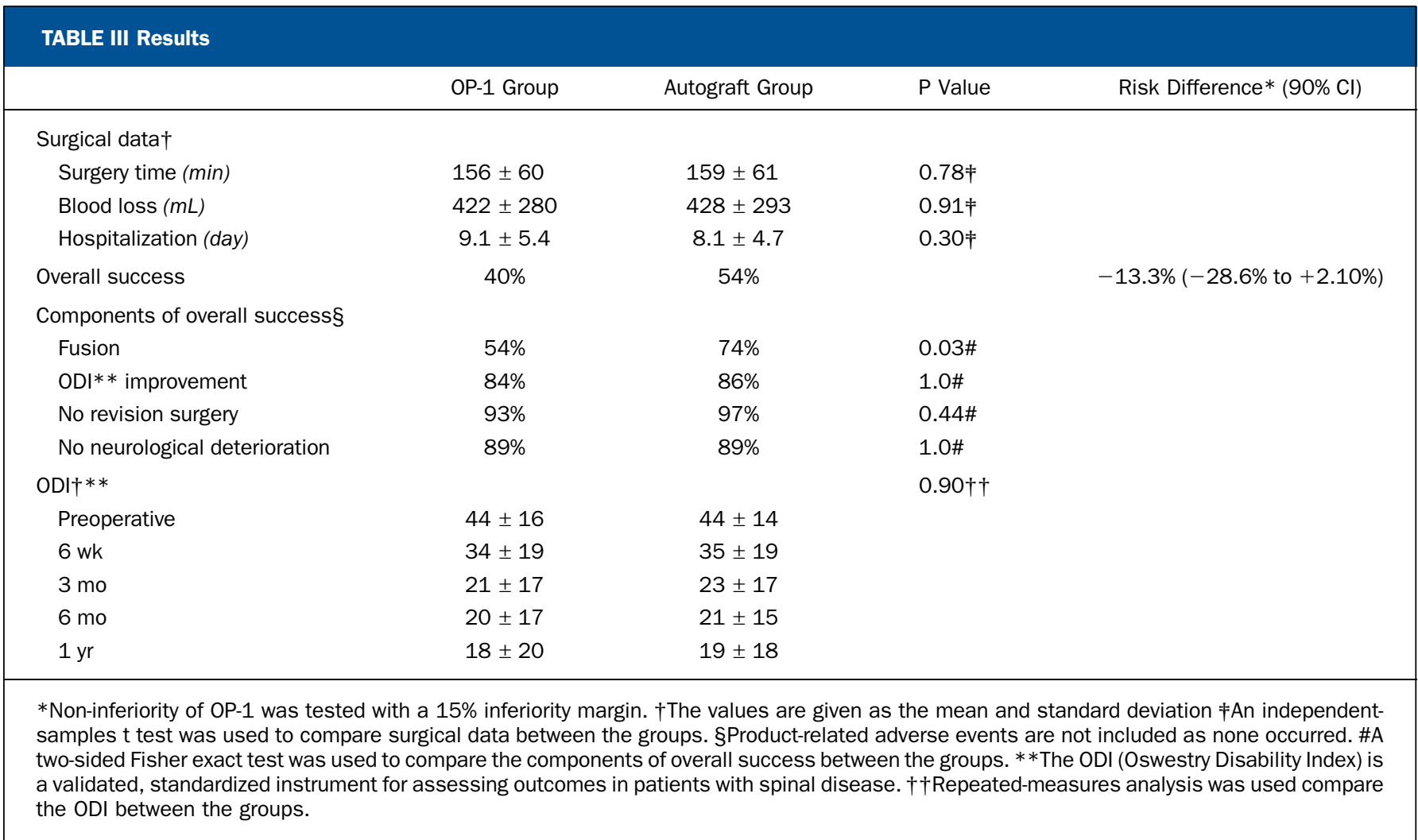

was registered in the ISRCTN registry (Current Controlled Trials number ISRCTN43648350) and was performed according to the principles of the Declaration of Helsinki and the Guideline for Good Clinical Practice ${ }^{15}$. We previously reported the results of a pilot study of safety and feasibility ${ }^{16}$.

The study, which involved nine participating centers in four European countries, included two treatment groups in a 1:1 ratio: one group received OP-1 combined with local bone (OP-1 group) and the other was treated with autologous bone graft from the iliac crest combined with local bone (autograft group). Patients were examined preoperatively and at six weeks, three months, six months, and twelve months after surgery. The primary outcome was defined as a combination of clinical outcome and radiographic evidence of fusion. The safety of OP-1 was evaluated by comparing the adverse events that occurred in both groups.

All patients had degenerative or isthmic spondylolisthesis with symptoms of neurological compression caused by central or foraminal stenosis that qualified for decompression and instrumented fusion of a single lumbar level. Table I shows the inclusion and exclusion criteria used in this study.

\section{Randomization}

A computer-generated randomization scheme with a permuted-block design was produced by an independent researcher using SYSTAT for Windows (SYSTAT). Each center received codes for an equal number of patients per treatment group. Randomization codes were stored in sealed opaque envelopes. The surgeons were blinded to the treatment group until decompression and placement of the pedicle screws were completed.

\section{Surgical Technique}

The surgical procedures, which were standardized during consensus meetings, were performed through a posterior midline approach. Transverse processes and facet joints were decorticated at the beginning of the procedure to obtain hemostasis before implantation. Decompression was achieved by complete or partial laminectomy and/or medial facetectomies. The same pedicle screw-rod instrumentation (Xia Spinal System; Stryker) was used in all patients.
In the OP-1 group, one unit of Osigraft containing $3.5 \mathrm{mg}$ of lyophilized recombinant human OP-1 in $1 \mathrm{~g}$ of type-I-collagen carrier was used on each side. Each unit was prepared in a separate bowl. Local bone derived from the decompression was morselized; was divided into the two bowls; and, after addition of $2.5 \mathrm{~mL}$ of fresh blood, was mixed with the Osigraft. To improve handling, the mixture was allowed to clot for a minimum of fifteen minutes before implantation. In the autograft group, bone was harvested from the iliac crest, morselized, and mixed with local autograft.

After a dry fusion bed was secured, the graft was placed on the decorticated osseous surfaces. The wound was closed without a deep drain. No nonsteroidal anti-inflammatory drugs were allowed for six weeks. Thrombosis prophylaxis was given according to local protocol. A brace or orthosis was worn for at least eight weeks postoperatively, in order to protect the spine from excessive movements. The type of brace was according to the local protocol and was not further specified.

\section{Radiographic Measurements}

Computed tomography (CT) scans obtained at one year postoperatively were reviewed by a spine surgeon and a radiologist blinded to the treatment and the institution. A third observer was consulted to adjudicate conflicting findings. If all three observers classified the fusion differently, the case was classified as "doubtful fusion." A system based on the Christensen $\operatorname{score}^{17}$ was used to assign one of three classifications:

1. Fusion: a continuous osseous bridge from the base of the pedicle and transverse processes connecting one vertebra to the other, at least on one side, in the absence of secondary signs of nonunion such as fracture or loosening of the screws or rods. If the fusion was doubtful in any way, the patient was not considered to have fusion.

2. Doubtful fusion: suboptimal quality of bone bridging or doubts about continuity on at least one side of the spine and an absence of fusion on the other side.

3. Nonunion: definite discontinuity or a lack of a fusion mass on both sides of the spine. 
The Journal of Bone \& Joint Surgery $\cdot$ JbJs.org Volume 98-A · Number 6 - March 16, 2016
OP-1 Compared With Iliac Crest Autograft in

Instrumented Posterolateral Fusion

\section{Clinical Measurements}

Clinical assessments were performed at all time points using the Oswestry Disability Index $(\mathrm{ODI})^{18}$. The patients were not blinded to the treatment group because of the occasional additional wound created by the iliac crest harvesting. Data on surgical time, number of days in the hospital, and blood loss were collected.

\section{Primary End Point}

The primary end point was overall success based on the twelve-month clinical and radiographic results. The result was considered a success when the patient had evidence of fusion on the CT scan, improvement in the ODI of $\geq 20 \%$ from baseline, no deterioration in neurological status, no additional surgical intervention to promote fusion, and no serious product-related adverse event.

\section{Safety Evaluation}

The safety of OP-1 was evaluated by documenting details and severity of all adverse events. An adverse event included any untoward medical occurrence, regardless of the nature of the event or its severity and without the need for a causal relationship with the treatment. Each event was evaluated for a relationship with the OP-1 treatment.

\section{Statistical Analysis}

Baseline characteristics were assessed by comparing means or percentages. Differences in confounding baseline characteristics were addressed with the appropriate analyses.

A non-inferiority margin of $15 \%$ was determined by setting it against the advantage of avoiding potential complications related to bone harvesting, the rate of which has been reported to be $8 \%$ to $41 \%^{3-5}$. The null hypothesis was that the degree of inferiority of OP- 1 to iliac crest autograft, based on overall success, was greater than the non-inferiority margin of $15 \%$ (success of autograft success of OP-1 $\geq 15 \%$ ). Non-inferiority was tested against the upper limit of a two-sided $90 \%$ confidence interval (CI), corresponding to a $5 \%$ test.

The sample size was determined on the basis of a success rate of $80 \%$ for iliac crest autograft, derived from data in the literature ${ }^{19-22}$. To obtain a power of $80 \%$ with an alpha of 0.10 , sixty-five patients were required in each group with use of a 1:1 randomization ratio.

As reoperations were considered failures in the primary overall outcome measurement, intention-to-treat analysis was similar to per-protocol analysis. Patients with missing data at one year were excluded from the primary analysis. The intention-to-treat principle was applied for secondary outcome measurements. A two-sided Fisher exact test was used to assess differences in all subcomponents of overall success between the OP-1 and autograft groups, and an independent-samples $t$ test was used to assess differences in surgery time, number of days in the hospital, and blood loss between the groups. Repeated-measures analysis was used to assess any significant differences in ODI scores between the groups. In cases of missing ODI scores, the last value was carried forward.

Data were collected by the University Medical Center Utrecht, processed using FileMaker Pro (FileMaker), and analyzed with SPSS 17.0 (IBM). Analysis of risk differences was performed with STATA/IC 11 (StataCorp). All values are given as the mean and standard deviation.

\section{Source of Funding}

The Department of Orthopaedics, University Medical Center Utrecht, received an unconditional grant from Stryker to partially fund this investigator-initiated

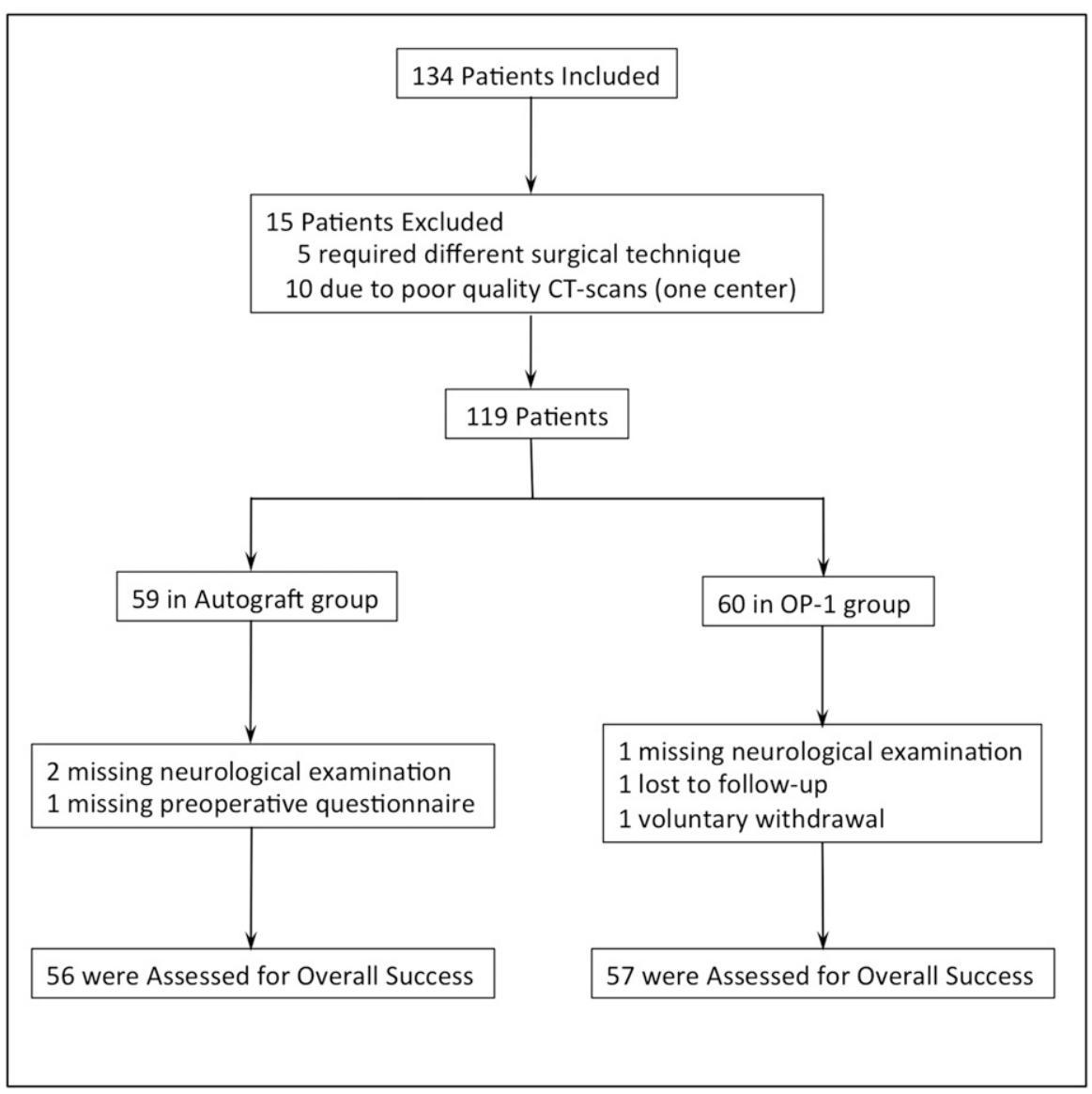

Fig. 1

Flow diagram for patients included in the analysis of overall success of OP-1 compared with iliac crest autograft. 


\begin{tabular}{|c|c|c|}
\hline & OP-1 Group (N = 57) & Autograft Group $(\mathrm{N}=56)$ \\
\hline Respiratory & $1(2 \%)$ & $0(0 \%)$ \\
\hline Gastrointestinal & $0(0 \%)$ & $0(0 \%)$ \\
\hline Dural tear & $3(5 \%)$ & $4(7 \%)$ \\
\hline \multicolumn{3}{|l|}{ Surgical infection } \\
\hline Superficial & $2(4 \%)$ & $2(4 \%)$ \\
\hline Deep & $3(5 \%)$ & $2(4 \%)$ \\
\hline Instrumentation failure & $2(4 \%)$ & $3(5 \%)$ \\
\hline Herniation of intervertebral disc & $0(0 \%)$ & $1(2 \%)$ \\
\hline Excessive back/leg pain & $3(5 \%)$ & $2(4 \%)$ \\
\hline Miscellaneous $\dagger$ & $3(5 \%)$ & $3(5 \%)$ \\
\hline
\end{tabular}

trial. The investigators own the data and were responsible for the protocol, data analysis, and interpretation. The study was monitored by an independent trial monitor who was hired by the investigators and reported to the investigators.

\section{Results}

The study originally included 134 patients. After exclusions (Fig. 1), the analysis of overall outcomes was based on 113 patients-fifty-seven in the OP-1 group and fifty-six in the autograft group.

The groups did not differ with regard to any baseline characteristics except for the percentage of reported smokers, which was $48 \%$ in the OP- 1 group and $31 \%$ in the autograft group (Table II). There were no differences in blood loss, surgery time, or duration of hospitalization between the groups (Table III).

The rate of overall success was $40 \%$ in the OP- 1 group and $54 \%$ in the autograft group (risk difference $=-13.3 \%, 90 \% \mathrm{CI}=$ $-28.6 \%$ to $+2.10 \%)$. Non-inferiority of OP-1 was thus not demonstrated. The difference in the overall success was caused by the significantly lower fusion rate as seen on CT in the OP-1 group: $54 \%$ versus $74 \%$ ( $95 \% \mathrm{CI}$ of difference $=-40.1 \%$ to $-2.98 \%, \mathrm{p}=0.03$ ).

Because of the differences in smoking rates between the groups, a multiple logistic regression analysis was performed. The overall outcome was not affected by smoking $(\mathrm{p}=0.52)$ or treatment group $(\mathrm{p}=0.15)$. The rate of fusion seen on CT was also not affected by smoking $(\mathrm{p}=0.90)$ but was significantly influenced by the treatment group $(\mathrm{p}=0.02)$, confirming the results of the primary analysis.
The radiographic classification in the OP-1 group was fusion in $54 \%$, doubtful fusion in $28 \%$, and nonunion in $18 \%$ compared with $74 \%, 10 \%$, and $16 \%$, respectively, in the autograft group. There were no significant differences $(p=0.90)$ in the mean ODI scores between the groups (Table III).

Twenty-three adverse events were reported in the OP-1 group compared with twenty-nine in the autograft group (Table IV). In one patient in the OP-1 group, a primary brain tumor was diagnosed eleven months after the surgery; this was considered unrelated to the treatment ${ }^{16}$. A reoperation was performed in ten patients in the OP-1 group and two patients in the autograft group. The indications for the reoperation in the OP-1 group were infection (four patients), instrumentation failure (two), neurological disturbances (three), and instrumentation removal due to residual back pain (one). The probable causes of the neurological disturbances were extrapedicular screw placement in one patient and a hematoma in another; no cause was found in the third patient. The indications for reoperation in the control group were infection and instrumentation failure. None of the adverse events could be directly related to the use of OP-1. No complications occurred in conjunction with the iliac crest autograft.

\section{Discussion}

T $\mathrm{n}$ what we believe is the first large multicenter, randomized 1 non-inferiority study comparing OP-1 with iliac crest autograft in single-level instrumented posterolateral spinal fusion, we 
The Journal of Bone \& Joint Surgery · JBJS. Org Volume 98-A · Number 6 - March 16, 2016
OP-1 Compared with Iliac Crest Autograft in

Instrumented Posterolateral Fusion found significantly lower fusion rates with OP-1 (54\% versus $74 \%, \mathrm{p}=0.03$ ). As a result of this lower rate of fusion seen on CT, non-inferiority of OP-1 was not demonstrated, despite similar clinical outcomes in the two groups.

Previous studies have also shown a lack of a clear relationship between osseous fusion and clinical results. However, since clinical outcomes may deteriorate within several years if intended osseous fusion is not achieved ${ }^{23}$, our follow-up period of one year may have been too short to show differences in clinical results. Since the purpose of the OP-1 was to create osseous fusion, we believe that an ability to do so at least as well as autograft is a minimal requirement. A potential advantage of OP-1 is shorter surgery time compared with that needed to perform iliac crest autografting. Although surgery time appeared to be similar between the two groups in our study, in the OP-1 group there was the delay of waiting for coagulation of the construct. This was a consequence of the experimental design, which required that the OP-1 mixture be prepared after the randomization was disclosed to the surgeon at the end of the procedure.

Previously reported disadvantages of using BMPs in the spine, such as ectopic bone formation, retrograde ejaculation, bone resorption, and soft-tissue swelling ${ }^{9}$, were not seen in the current study. However, more patients had a reoperation in the OP-1 group (ten versus two in the autograft group). Although this difference could not be directly related to specific problems associated with BMPs, it should be noted in the light of previous discussions of higher complication and reoperation rates with the use of BMP-2 $2^{11,12}$.

The clinical outcomes were similar in the two treatment groups, despite avoidance of bone harvesting in the OP-1 group. Since the reported prevalence of donor-site pain ranges from $8 \%$ to $41 \%{ }^{3-5}$, use of OP-1 instead of iliac crest bone graft could have been expected to have some effect on the clinical outcome, at least in the short term. However, previous trials of BMPs also failed to show a positive effect from the avoidance of iliac crest bone harvest ${ }^{24}$. A possible explanation may be overestimation of donor-site pain due to the inability of patients to differentiate between residual low-back pain, and donor-site morbidity ${ }^{3}$.

In addition to the pilot study preceding the current study ${ }^{16}$, there have been three previous randomized prospective studies of the use of OP-1 in posterolateral spine fusion. Johnsson et al..$^{25}$ and Kanayama et al. ${ }^{26}$ found that fusion rates in patients treated with OP-1 were lower than those in patients treated with autograft, but this difference was not statistically significant. Although their findings were interesting with regard to safety and feasibility, no conclusion can be made with regard to the effectiveness of OP-1 because of the limited numbers of patients (nine and ten). In the only other large study of OP-1 for spinal fusion, which in that trial was done without instrumentation, the authors concluded that OP-1 Putty was a safe and effective alternative to autograft ${ }^{27}$. However, they also failed to show non-inferiority of OP-1 based on an overall success score that included radiographic findings. As was the case in our study, the low overall success was caused by a significantly lower fusion rate. When subsequent, additional CT scans were obtained at more than three years postoperatively, they again showed a lower fusion rate in the OP-1 group (56\% versus $83 \%, \mathrm{p}=0.001$ ). However, with a modified definition of radiographic evidence of success (presence of new bone on CT scans instead of bridging bone, in combination with limited motion on dynamic radiographs), non-inferiority was demonstrated, which led to their conclusion that OP-1 was effective.

Our study is not completely comparable with previous studies. We used Osigraft, which is available in Europe and differs in composition from OP-1 Putty, which is used in the U.S. The two preparations contain the same amount of the active ingredient, OP-1, which is in the same collagen carrier. The only difference is the carboxymethylcellulose additive in the OP-1 Putty, which improves the handling characteristics. In the present study, we added fresh blood to the OP-1, which was allowed to clot to give it a more putty-like structure. We also mixed the OP-1 with local bone obtained from the decompression, a realistic scenario for BMP application as the availability of some local autograft is inherent to the surgical technique. Although local autograft alone can achieve spinal fusion, its efficacy compared with iliac crest autograft remains questionable in the absence of prospective studies ${ }^{28,29}$. Finally, patients with degenerative spondylolisthesis as well as those with isthmic spondylolisthesis were enrolled in the present study. This was done to make the conclusion relevant to a larger patient group with single-level low-grade spondylolisthesis requiring fusion surgery. Since instrumentation was used, the biomechanical differences were not expected to have a substantial effect on the fusion rates. The etiology of the instability might be of relevance to clinical outcomes but not to fusion rates. In addition, there was no difference in the distribution of the types of spondylolisthesis between the OP-1 and control groups.

There are several limitations to our study. Despite proper randomization, the percentage of smokers was higher in the OP-1 group (48\% versus 31\%). Smoking was not an exclusion criterion as we wanted the study series to represent the general patient population. Furthermore, OP-1 is specifically promoted for use in compromised patients, including smokers, as specified in the Humanitarian Device Exemption ${ }^{8}$. Moreover, several animal studies indicated that the inhibitory effect of smoking is overcome by using OP-1 instead of autograft ${ }^{30,31}$. In our study, smoking was not found to have an effect on overall outcome or radiographic evidence of fusion. The percentage of patients who were treated with an L5-S1 fusion was higher in the OP-1 group $(45 \%)$ than in the control group $(31 \%)$. We are not aware of published studies comparing fusion rates at different lumbar levels. Since our study involved instrumented fusion, the influence of the biomechanical differences is expected to be minimal. However, the distance between the transverse processes, and thereby the distance that needs to be bridged, might be smaller at the L5-S1 level compared with the other levels fused in our study. Although we do not expect this to be of major influence on fusion rates, this difference should be taken into account. Another limitation of our study was that the patients were not blinded to the treatment type because of the occasional additional wound for the iliac crest harvest. However, the surgeon 
The Journal of Bone \& Joint Surgery $\cdot$ JbjS. org Volume 98-A · Number 6 - March 16, 2016
OP-1 Compared with Iliac Crest Autograft in

Instrumented Posterolateral Fusion was blinded until the graft type was disclosed, and the observers who assessed the radiographic data were blinded to the treatment group. Finally, there were missing data, largely as a result of inadequate CT quality in one center, and this resulted in a lower number of patients than required according to the power calculation. Although this could have influenced the overall outcome assessment, it did not preclude the conclusion of a significantly lower fusion rate in the OP-1 group.

BMP use has multiplied severalfold during the last decade to more than 100,000 cases annually in the U.S. ${ }^{32}$. Recent publications have expressed concerns regarding the structural underreporting of serious product-related adverse events and substantial design biases of industry-sponsored BMP-2 trials $^{10-12,14,33}$. Whether different BMPs vary with regard to their effectiveness and complication rates in clinical applications is not known. Because of the potential complications and the lack of evidence of efficacy, caution is warranted when using BMPs for off-label spinal indications. Adequate clinical trials need to clearly define the safety profiles and efficacy of each kind of BMP product as well as the appropriate dosages and specific carriers before these compounds can be applied in spinal settings.

On the basis of the results of the current study, use of OP-1 in place of autologous iliac crest bone in instrumented posterolateral lumbar fusions cannot be recommended.
Wouter J.A. Dhert, $\mathrm{MD}, \mathrm{PhD}^{1}$

F. Cumhur Oner, $\mathrm{MD}, \mathrm{PhD}^{1}$

${ }^{1}$ Department of Orthopaedics, University Medical Center Utrecht, Utrecht, the Netherlands

${ }^{2}$ Department of Neurosurgery, Leiden University Medical Center, Leiden, the Netherlands

${ }^{3}$ Department of Orthopaedics, Rijnstate, Arnhem, the Netherlands

${ }^{4}$ Department of Orthopaedics, Hôpital Beaujon, Clichy, France

${ }^{5}$ Department of Orthopaedics and Traumatology, Ospedale di Circolo, Varese, Italy

${ }^{6}$ Department of Orthopaedics, Università Politecnica delle Marche, Ancona, Italy

${ }^{7}$ Department of Neurosurgery,

Hôpital La Tronche,

Grenoble, France

${ }^{8}$ Department of Orthopaedics, Jeroen Bosch Ziekenhuis, Den Bosch, the Netherlands

${ }^{9}$ Department of Orthopaedic Surgery and Traumatology, Hospital Clinico San Carlos, Madrid, Spain

${ }^{10}$ Department of Orthopaedic Surgery and Traumatology, Hospital Costa del Sol,

Marbella, Spain

${ }^{11}$ Department of Radiology,

Haaglanden Medical Center,

the Hague, the Netherlands

E-mail address for F.C. Oner: f.c.oner@umcutrecht.nl

\section{References}

1. Deyo RA, Nachemson A, Mirza SK. Spinal-fusion surgery - the case for restraint. N Engl J Med. 2004 Feb 12;350(7):722-6.

2. Arrington ED, Smith WJ, Chambers HG, Bucknell AL, Davino NA. Complications of iliac crest bone graft harvesting. Clin Orthop Relat Res. 1996 Aug;329:300-9.

3. Delawi D, Dhert WJ, Castelein RM, Verbout AJ, Oner FC. The incidence of donor site pain after bone graft harvesting from the posterior iliac crest may be overestimated: a study on spine fracture patients. Spine (Phila Pa 1976). 2007 Aug 1;32 (17):1865-8.

4. Fernyhough JC, Schimandle JJ, Weigel MC, Edwards CC, Levine AM. Chronic donor site pain complicating bone graft harvesting from the posterior iliac crest for spinal fusion. Spine (Phila Pa 1976). 1992 Dec;17(12):1474-80.

5. Younger EM, Chapman MW. Morbidity at bone graft donor sites. J Orthop Trauma. 1989;3(3):192-5.

6. Urist MR. Bone: formation by autoinduction. Science. 1965 Nov 12;150 (3698):893-9.

7. FDA. Approval letter for BMP-2. http://www.accessdata.fda.gov/cdrh_docs/pdf/ P000058a.pdf. 2002 Jul 2. Accessed 2015 Oct 22.

8. FDA. HDE approval letter for OP-1 in posterolateral fusion. http://www. accessdata.fda.gov/cdrh_docs/pdf2/H020008a.pdf. 2004 Jul 4. Accessed 2015 Oct 22.
9. Cahill KS, Chi JH, Day A, Claus EB. Prevalence, complications, and hospital charges associated with use of bone-morphogenetic proteins in spinal fusion procedures. JAMA. 2009 Jul 1;302(1):58-66.

10. Mitka M. Questions about spine fusion product prompt a new process for reviewing data. JAMA. 2011 Sep 28;306(12):1311-2.

11. Carragee EJ, Ghanayem AJ, Weiner BK, Rothman DJ, Bono CM. A challenge to integrity in spine publications: years of living dangerously with the promotion of bone growth factors. Spine J. 2011 Jun;11(6):463-8.

12. Carragee EJ, Hurwitz EL, Weiner BK. A critical review of recombinant human bone morphogenetic protein-2 trials in spinal surgery: emerging safety concerns and lessons learned. Spine J. 2011 Jun;11(6):471-91.

13. Branch CL Jr. Physican-directed (off-label) use of recombinant bone morphogenic protein-2: let us do it well! Spine J. 2011 Jun;11(6):469-70.

14. Spengler DM. Resetting standards for sponsored research: do conflicts influence results? Spine J. 2011 Jun;11(6):492-4.

15. Guideline for good clinical practice. http://www.ema.europa.eu/docs/en GB/ document_library/Scientific_guideline/2009/09/WC500002874.pdf. 2002 Jul 1. Accessed 2015 Oct 22.

16. Delawi D, Dhert WJ, Rillardon L, Gay E, Prestamburgo D, Garcia-Fernandez C, Guerado E, Specchia N, Van Susante JL, Verschoor N, van Ufford HM, Oner FC. A 
The Journal of Bone \& Joint Surgery $\cdot$ JBJS. Org Volume 98-A · Number 6 - March 16, 2016
OP-1 Compared With Iliac Crest Autograft in

Instrumented Posterolateral Fusion prospective, randomized, controlled, multicenter study of osteogenic protein-1 in instrumented posterolateral fusions: report on safety and feasibility. Spine (Phila Pa 1976). 2010 May 20;35(12):1185-91.

17. Christensen FB, Laursen M, Gelineck J, Eiskjaer SP, Thomsen K, Bünger CE. Interobserver and intraobserver agreement of radiograph interpretation with and without pedicle screw implants: the need for a detailed classification system in posterolateral spinal fusion. Spine (Phila Pa 1976). 2001 Mar 1;26(5):538-43; discussion 543-4

18. Fairbank JC, Couper J, Davies JB, O'Brien JP. The Oswestry low back pain disability questionnaire. Physiotherapy. 1980 Aug;66(8):271-3.

19. An HS, Lynch K, Toth J. Prospective comparison of autograft vs. allograft for adult posterolateral lumbar spine fusion: differences among freeze-dried, frozen, and mixed grafts. J Spinal Disord. 1995 Apr;8(2):131-5.

20. Greenough CG, Peterson MD, Hadlow $S$, Fraser RD. Instrumented posterolatera lumbar fusion. Results and comparison with anterior interbody fusion. Spine (Phila Pa 1976). 1998 Feb 15;23(4):479-86.

21. Thalgott JS, Sasso RC, Cotler HB, Aebi M, LaRocca SH. Adult spondylolisthesis treated with posterolateral lumbar fusion and pedicular instrumentation with AO DC plates. J Spinal Disord. 1997 Jun;10(3):204-8.

22. Thomsen K, Christensen FB, Eiskjaer SP, Hansen ES, Fruensgaard S, Bünger CE. 1997 Volvo Award winner in clinical studies. The effect of pedicle screw instrumentation on functional outcome and fusion rates in posterolateral lumbar spinal fusion: a prospective, randomized clinical study. Spine (Phila Pa 1976). 1997 Dec 15;22(24):2813-22.

23. Fischgrund JS. The argument for instrumented decompressive posterolateral fusion for patients with degenerative spondylolisthesis and spinal stenosis. Spine (Phila Pa 1976). 2004 Jan 15;29(2):173-4

24. Carragee EJ, Bono CM, Scuderi GJ. Pseudomorbidity in iliac crest bone graft harvesting: the rise of rhBMP-2 in short-segment posterior lumbar fusion. Spine J. 2009 Nov;9(11):873-9.

25. Johnsson $R$, Strömqvist $B$, Aspenberg $P$. Randomized radiostereometric study comparing osteogenic protein-1 (BMP-7) and autograft bone in human noninstrumented posterolateral lumbar fusion: 2002 Volvo Award in clinical studies. Spine (Phila Pa 1976). 2002 Dec 1;27(23):2654-61.
26. Kanayama M, Hashimoto $T$, Shigenobu K, Yamane S, Bauer TW, Togawa D. A prospective randomized study of posterolateral lumbar fusion using osteogenic protein-1 (OP-1) versus local autograft with ceramic bone substitute: emphasis of surgical exploration and histologic assessment. Spine (Phila Pa 1976). 2006 May 1;31(10):1067-74.

27. Vaccaro AR, Lawrence JP, Patel T, Katz LD, Anderson DG, Fischgrund JS, Krop J, Fehlings MG, Wong D. The safety and efficacy of OP-1 (rhBMP-7) as a replacement for iliac crest autograft in posterolateral lumbar arthrodesis: a long-term (>4 years) pivotal study. Spine (Phila Pa 1976). 2008 Dec 15;33 (26):2850-62.

28. Sengupta DK, Truumees E, Patel CK, Kazmierczak C, Hughes B, Elders G, Herkowitz HN. Outcome of local bone versus autogenous iliac crest bone graft in the instrumented posterolateral fusion of the lumbar spine. Spine (Phila Pa 1976). 2006 Apr 20;31(9):985-91.

29. Inage $K$, Ohtori $S$, Koshi $T$, Suzuki M, Takaso M, Yamashita M, Yamauchi K, Inoue G, Orita S, Eguchi Y, Ochiai N, Kishida S, Kuniyoshi K, Aoki Y, Nakamura J, Ishikawa T, Arai G, Miyagi M, Kamoda H, Suzuki T, Toyone T, Takahashi K. One, two-, and three-level instrumented posterolateral fusion of the lumbar spine with a local bone graft: a prospective study with a 2-year follow-up. Spine (Phila Pa 1976). 2011 Aug 1;36(17):1392-6.

30. Patel TC, Erulkar JS, Grauer JN, Troiano NW, Panjabi MM, Friedlaender GE. Osteogenic protein-1 overcomes the inhibitory effect of nicotine on posterolateral lumbar fusion. Spine (Phila Pa 1976). 2001 Aug 1;26(15):1656-61.

31. Grauer JN, Vaccaro AR, Kato M, Kwon BK, Beiner JM, Patel TC, Hilibrand AS, Chiba K, Albert TJ. Development of a New Zealand white rabbit model of spinal pseudarthrosis repair and evaluation of the potential role of OP-1 to overcome pseudarthrosis. Spine (Phila Pa 1976). 2004 Jul 1;29(13): 1405-12.

32. Ong KL, Villarraga ML, Lau E, Carreon LY, Kurtz SM, Glassman SD. Off-label use of bone morphogenetic proteins in the United States using administrative data. Spine (Phila Pa 1976). 2010 Sep 1;35(19):1794-800.

33. Mirza SK. Folly of FDA-approval studies for bone morphogenetic protein. Spine J. 2011 Jun;11(6):495-9.

34. Meyerding HW. Spondylolisthesis. Surg Gynecol Obstet. 1932;54:371-7. 